\title{
OBSERVADA LA "PARTICULA DE DIOS" EN EL LHC
}

\author{
Hernando Gonzalez Sierra \\ Doctor en Fisica. Facultad de Ciencias Exactas y Naturales \\ Universidad Surcolombiana. hergosi@usco.edu.co \\ Ricardo Gaitan Lozano \\ Doctor en Fisica. Facultad de Ciencias Exactas y Naturales \\ Universidad Surcolombiana. rgaitan@usco.edu.co \\ Jose Halim Montes De Oca Yemha \\ Doctor en Física. Facultad de Estudios Superiores Cuautitlán \\ Universidad Nacional Autónoma de México. josealhim@gmail.com
}

\begin{abstract}
Resumen
En este artículo presentamos un análisis y conse-cuencias de los resultados acerca de la búsqueda del Bosón de Higgs en el laboratorio más grande de altas energías, el LHC o gran colisionador de Hadrones que funciona en el Centro Europeo de Investiga-ciones Nucleares CERN. El bosón de Higgs, más comúnmente llamado "la partícula divina" es el responsable del mecanismo que genera la masa dentro del contexto de Modelo Estándar de la Física de partículas elementales
\end{abstract}

Palabras clave: Modelo Estándar, masa, colisión, Física.

\section{Introducción}

Después de 5 años de funcionamiento del LHC [1] , Gran Colisionador de Hadrones, ubicado en la frontera entre Francia y Suiza, la organización del ICHEP 2012 (Congreso Internacional de Física de Altas Energías) [2] , que se celebra en Melbourne (Australia), ha informado el dí 4 de julio, que se observó lo que podría ser el bosón de Higgs con una masa de 125.3 Gev [3], en unidades de energía, con un nivel de certeza del $99.99994 \%$ que equivale a un error de 1 parte en 2 millones.

El experimento consiste en hacer colisionar dos haces de protones (Hadrones), que se mueven en direcciones contrarias, con velocidades cercanas a la velocidad de propagación de la luz en el espacio vacío [4] ; para obtener señales de nuevas partículas en rangos de energía no explorados anteriormente. Los protones son acelerados por campos eléctricos y magnéticos en un recorrido circular de $27 \mathrm{~km}$ de diámetro. Con el descubrimiento del bosón de Higgs se empieza a aclarar el enigma "del origen la masa" en el modelo estándar.

El apelativo de " partícula de Dios" fue acuñado por el premio Nobel de Física León Lederman [5]. El bosón de Higgs es el último eslabón que le falta al modelo estándar de la física de partículas elementales [6] puesto que las restantes partículas predichas por el modelo ya fueron encontradas.

El modelo estándar de la Física de partículas elementales es una teoría de norma [7], que unifica tres interacciones de la naturaleza: La electromagnética, la fuerte nuclear y la débil nuclear. La interacción gravitacional, la más conocida y que nos afecta cotidianamente, no forma parte del modelo estándar ya que no se ha logrado unificar con las otras tres interacciones, debido a incompatibilidades matemáticas.

En el modelo estándar las tres interacciones tienen manifestaciones similares: Están mediadas por 
intercambio de partículas portadores de fuerza, los llamados bosones vectoriales y están protegidas por una simetría llamada "simetría de norma". El mediador, o portador, de la interacción electromagnética es el fotón, la interacción débil nuclear tiene tres portadores de fuerza que son los dos bosones W (uno con carga eléctrica positiva y el otro con carga eléctrica negativa) y el bosón $Z$ (sin carga eléctrica, como el fotón). En la figura 1 se ilustra un proceso que da lugar a la producción del bosón de Higgs, mediante una representación pictórica comúnmente usada por los físicos de partículas denominada Diagrama de Feynman [8].

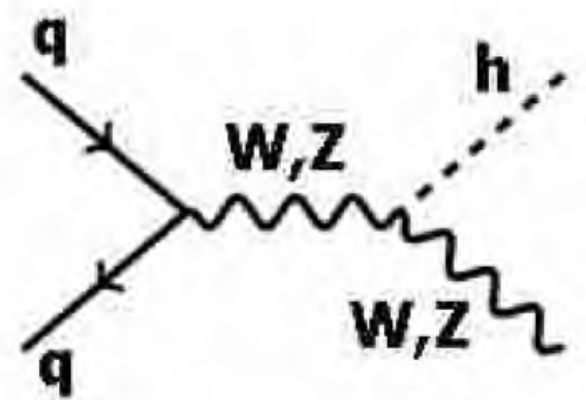

Figura 1: Diagrama de Feynman de un posible canal para producir el bosón de Higgs. La colisión de un par quark-antiquark (componentes de los Hadrones) produce un bosón vectorial ( $W \circ Z$ ) que emite la "partícula de Dios", representada por la linea punteada y denotada con la letra $h$. Tomado de http://www.taringa.net/posts/info/10064170/Elboson-de-Higgs-se-hace-el-dificil.html

\section{Las interacciones fundamentales}

La interacción electromagnética es producida por efectos de cargas eléctricas y corrientes eléctricas [9]; la interacción débil [10] ocurre a pequeñas distancias, en el interior de los núcleos atómicos, y es responsable de procesos como el decaimiento beta [11] en donde un protón es trasformado en un electrón , un neutrón y un antineutrino. Después del decaimiento beta el núcleo atómico en el que tiene lugar el proceso se transmuta en otro nuevo. En la interacción nuclear fuerte, que ocurre al interior de los núcleos atómicos, los mediadores son los gluones [12] ; estas ultimas partículas no permiten a los componentes de los protones y neutrones, los quarks, permanecer en estados libres.
La unificación de las tres interacciones en el modelo estándar no es completa. En términos matemáticos esto indica que no tenemos un solo grupo de norma, sino tres grupos de norma llamados SU(3), para la interacción fuerte, SU(2) , para la interacción débil y $\mathrm{U}(1)$, para la interacción electromagnética. La matemática inherente a las teorías de norma de la Física de partículas elementales es la teoría de grupos. La unificación en el modelo estándar no es completa porque sólo la interacción electromagnética y la débil han sido unificadas en el grupo SU(2)xU(1) (Modelo Estándar Electro-débil) [13], quedando la interacción fuerte independientemente descrita por el grupo $S U(3)$.

La Física de partículas elementales es un ingrediente en la explicación de la teoría del Big-Bang (Gran Explosión) [14] , teoría científica sobre el origen del universo. En el instante que se formó el universo las cuatro interacciones estaban unificadas y no existía ninguna diferencia entre ellas. A medida que el universo se fue enfriando, las manifestaciones de cada una de las interacciones se hicieron notables y se fueron diferenciando entre si. El proceso mediante el cual las interacciones se hacen diferentes, separándose una de otra, se denomina "Ruptura espontánea de la simetría" [15] .

Según la teoría del Big-Bang, una billonésima de segundo después de ocurrida la gran explosión, cuando la temperatura del universo era cercana a los 100000 millones de grados kelvin, se produjo la ruptura espontánea de la simetría electro-débil (separación de la interacción electromagnética de la débil). Las partículas que carecían de masa, antes de la ruptura espontánea de la simetría, adquieren masa.

\section{El bosón de Higgs y la ruptura espontanea de la simetría}

El campo de Higgs es el responsable, en el modelo estándar electro-débil, de la ruptura espontánea de la simetría SU(2)xU(1). Parece complicado el asunto, pero no lo es. Veamos : Además de los campos que conocemos como campo electromagnético y campo gravitacional, el Físico Peter Higgs en 1964 introdujo un campo de energía que lleva su nombre , para dar una explicación al hecho de que después de la ruptura espontánea de la simetría algunas partículas que conforman el grupo $\mathrm{SU}(2) \times \mathrm{U}(1)$, quedando 
como remanente el grupo $U(1)$, adquieren masa. Después del proceso, llamado "Mecanismo Higgs", etapa en la cual uno de los componentes del campo de Higgs desarrolla un valor de expectación del vacío, los fermiones y los portadores de la interacción débil adquieren masa. Los fermiones son los 6 quarks, el electrón, el muón, y el tauón, estas dos últimas partículas tienen la misma carga eléctrica del electrón pero son más pesadas e inestables.

No es exacto que el bosón de Higgs de masa a otras partículas: Lo que ocurre es el efecto de una interacción, entre el campo escalar de Higgs y partículas sin masa, adquiriendo estas ultimas sus masas por acción de esa interacción. Los fotones y gluones no tienen masas debido a que ellos no son influenciados por la interacción, caso contrario ocurre a los fermiones y bosones intermediarios que adquieren masa por ese mecanismo ideado por Peter Higgs.

Las partículas elementales se diferencian por una propiedad intrínseca llamada "espín". Las partículas con espín semientero $(1 / 2,3 / 2,5 / 2, \ldots)$ se denominan "fermiones" y las partículas con espín entero $(0,1,2,3 \ldots)$ se llaman "bosones". El espín es un número cuántico; en el caso de los electrones, que son fermiones, proporciona una explicación de la diversidad de elementos existentes en la naturaleza mediante el principio de exclusión de Paulí.

El descubrimiento del bosón de Higgs permite iniciar la búsqueda del esclarecimiento de otros enigmas relacionados con la Física de las partículas elementales como lo son: la materia oscura [16], la energía oscura [17], dimensiones extras [18], mundos paralelos [19], oscilaciones de los neutrinos [20], Supersimetría [21], asimetría materia-anti materia [22].

De acuerdo con los últimos datos reportados por el CERN parece muy probable que la partícula encontrada sea el buscado bosón de Higgs. Los análisis realizados están basados en los resultados obtenidos en los experimentos ATLAS y CMS del LHC. Ellos han visto indicios de la presencia del bosón de Higgs en la región comprendida entre los 124 y $126 \mathrm{GeV}$.

\section{Conclusiones}

La búsqueda del bosón de Higgs en el gran colisionador de hadrones ha mostrado la presencia de eventos que podrían permitir su clara detección en las próximos experimentos del CERN que continuaran en el año 2014. Los resultados aunque alentadores no son definitivos, pero se prevé que se está muy cerca de poder dilucidar cual es el origen de la masa.

Otros resultados que se buscan en los detectores del LHC están relacionados con dimensiones extras del espacio, diferentes a las que ya conocemos, la posible existencia de partículas supersimétricas, los enigmas de la materia oscura y de la asimetría bariónica del universo.

\section{Referencias Bibliográficas}

[1] http://public.web.cern.ch/pubiic/en/hhc/hc-en.htmILHC

[2] XXVI Conferencia Internacional de Física de Altas Energías, celebrado en Melbourne (Australia) en 2012.

[3] J. E Gunion et al. The Higgs Hunter's Guide. Frontiers in physics, 1990

[4] P.A Tilpler y .Mosca. Física para la ciencia y tecnologia: Luz. Editorial Reverte, 2005

[5] L. Lederman. The god particle: If the universe is the answer, what is the question. Penguin books, 1964

[6] S Weinberg. The Quantum Theory of Fields, volume II. Cambridge University Press, 1995

[7] I.J.R Aitchison and A.J.G Hey. Gauge Theories in Particle Physics. Taylor \& Francis Group, 2003.

[8] J.J Sakurai. Advanced Quantum Mechanics.Addison Wesley Publishing, 1967

[9] Levich. Mecánica Cuantica. Editorial Reverté, 2005.

[10] J.C Taylor. Gauge Theories of Weak Interactions. Cambridge University Press, 1973

[11] R.A Serway y J.W Jewett. Editorial Thomson, 2011.

[12] R. Penrose. Lo grande , lo pequeño y la mente humana. Cambridge University Press, 2005.

[13] R.E Martinez. Teoría Cuántica de Campos. Universidad Nacional de Colombia, 2007 
[14] C.C Radovic. ¿Porqué ocurrió el big-bang? El enigma del origen del universo. Editorial Universitaria, 2005

[15] W. Thirring. Impresiones Cósmicas: $L$ as huellas de Dios en las leyes de la naturaleza. Libros de zorzal, 2008

16] T. Matos. ¿De qué está hecho el universo? Materia oscura y energía oscura. Ciencia para todos, 2004.

[17] ibid [16]

[18] L. Stewart. El big-bang del génesis. Bubok publishing, 2008

[19] Ibid [18]

[20] A. Ferrer y E. Ros . Física de partículas y Astro-partículas. Universidad de Valencia, 2005

[21] J. Gribbin. Supersimetría, cuerdas y teoría del todo. Drakontos, 2007

[22] C Chardin . La antimateria. Siglo XXI editores, 2001. 